\title{
An Autoethnography: Outcomes from Faculty Engagement in Course Development in a Large First-Year Engineering Program
}

\section{Dr. Holly M Matusovich, Virginia Tech}

Dr. Holly M. Matusovich is an Associate Professor in the Department of Engineering Education. She is current the Assistant Department Head for Undergraduate Programs and the former Assistant Department Head for Graduate Programs in Virginia Tech's Department of Engineering Education. Dr. Matusovich is recognized for her research and practice related to graduate student mentoring. She won the Hokie Supervisor Spotlight Award in 2014, was nominated for a Graduate Advising Award in 2015, and won the 2018 Graduate Student Mentor Award for the College of Engineering. Dr. Matusovich has graduated 10 doctoral students since starting her research program in Spring 2009. Dr. Matusovich co-hosts the Dissertation Institute, a one-week workshop each summer funded by NSF, to help underrepresented students develop the skills and writing habits to complete doctorate degrees in engineering. Across all of her research avenues, Dr. Matusovich has been a PI/Co-PI on 12 funded research projects including the NSF CAREER Award with her share of funding be ingnearly $\$ 2.3$ million. She has co-authored 2 book chapters, 21 journal publications and more than 70 conference papers. She has won several Virginia Tech awards including a Dean's Award for Outstanding New Faculty, an Outstanding Teacher Award and a Faculty Fellow Award. She holds a B.S. in Chemical Engineering from Cornell University, an M.S. in Materials Science from the University of Connecticut and a Ph.D. in Engineering Education from Purdue University.

\section{Dr. Homero Murzi, Virginia Polytechnic Institute and State University}

Homero Murzi is an Assistant Professor in the Department of Engineering Education at Virginia Tech. He holds degrees in Industrial Engineering (BS, MS), Master of Business Administration (MBA) and in Engineering Education $(\mathrm{PhD})$. Homero has 15 years of international experience working in industry and academia. His research focuses on contemporary and inclusive pedagogical practices, industry-driven competency development in engineering, and understanding the barriers that Latinx and Native Americans have in engineering. Homero has been recognized as a Diggs scholar, a Graduate Academy for Teaching Excellence fellow, a Diversity scholar, a Fulbright scholar and was inducted in the Bouchet Honor Society.

\section{Dr. David Gray, Virginia Tech}

Dr. Gray receieved his B.S. in Electrical and Computer Engineering from Virginia Tech in 2000. He then earned a M.S. and a Ph.D. in Materials Science and Engineering from Virginia Tech in 2002 and 2010, respectively. Much of his graduate education focused on semiconductor devices physics and materials processing. However, his actual Ph.D. dissertation was on thermal modeling and process control of a friction stir fabrication method of additive manufacturing. Dr. Gray followed up his Ph.D. with a position as a post-doctoral associate under the guidance of Dr. Dwight Veihland working with composite magnetic field sensors. After his education, Dr. Gray continued his research in small-business environments, developing technologies and products across a wide range of fields including magnetic materials, sensors, and devices, energy harvesting technologies, harsh environment sensing, additive manufacturing, non-destructive inspection and evaluation, and vehicle autonomy. Dr. Gray came to the Engineering Education department as an instructor in 2018, and was promoted to Associate Professor of Practice in August 2019. Dr. Gray is primarily focused on pedagogy of first-year engineering students, but maintains an undergraduate research group with interests in automotive systems, communications, computing, and non-destructive inspection.

\section{Dr. Benjamin D Chambers, Virginia Tech Department of Engineering Education}

Benjamin Chambers is an Associate Professor of Practice in the Department of Engineering Education at Virginia Tech. He is an interdisciplinary scholar with three degrees from Virginia Tech, including an MS Civil Infrastructure Engineering, MS Entomology, and a PhD in Environmental Design and Planning. Ben also has professional experience in utility-scale wind power development. His educational research 
interests include student creativity and mindfulness, as well as the built environment as an educational tool for engineering, biology, and ecology. He is also interested in animal interactions with buildings, particularly overwintering stink bugs.

\section{Matthew B James P.E., Virginia Tech}

Matthew James is an Assistant Professor of Practice in Engineering Education at Virginia Tech, and is a registered Professional Engineer in the State of Virginia. He holds bachelors and masters degrees from Virginia Tech in Civil Engineering. 


\title{
An Autoethnography: Outcomes from Faculty Engagement in Course Development in a Large First-Year Engineering Program
}

\begin{abstract}
This complete evidence-based practice documents recommendations associated with the planning and implementation process used by one institution in redeveloping its first-year general engineering courses. Using an autoethnographic approach, we share and critically examine our process for revamping the core courses (Foundations of Engineering I and Foundations of Engineering II) over the past two years. Suggestions based on our findings are provided for practitioners, instructional designers, and administrators.
\end{abstract}

\section{Introduction}

Considerable research focuses on the course structures, course content, and specific teaching practices within first year courses and programs. However, much less research focuses on the development of such courses and programs and how they evolved to the current state. One of the few studies that reported this process was conducted by Froyd and Rogers [1] in the 90's that analyzes the process of development and creation of a first year engineering program, and there have not been more significant studies since then that focus on first year engineering programs. We consider that research describing the process of development and improvement of first year programs is important and necessary in engineering education because of the fast-paced advancement of the engineering field. First year general engineering programs are widely implemented and are designed to help students transition into engineering programs and to develop engagement with the engineering field, to develop foundational technical and professional skills, and to help students select their engineering major [2]. First year programs are continuously changing to effectively serve their main stakeholders. For example, engineering departments have high expectations of how first year programs will prepare their students. Similarly, first year programs need to understand current trends in the engineering field to be able to develop relevant content to engage students. Hence, understanding how such programs are improved and redesigned becomes crucial so other engineering educators in charge of these processes can take full advantage of lessons learned in course development or redevelopment across the field of engineering education.

To begin closing the gap in the literature about the process of course development, we undertook an autoethnography with the goal of providing advice from first-hand experience to assist in the implementation of similar processes at other institutions or in different fields. We answered the research question: What are the individual, collective, and pragmatic outcomes from engaging faculty from a variety of backgrounds in on-going continual course development and improvement in a large first-year engineering program? For purposes of this analysis, we consider the outcomes to be recommendations we would make to others because they represent the tangible and transferable outcomes. Autoethnography is a research methodology that analyzes a phenomenon through the use of self-narratives, which would otherwise remain private or buried [3]. This approach enables us to share the combined but individual experiences of the 
professors of practice that completed the curriculum restructuring situated within the context of work.

\section{Theoretical Framework}

Our study is guided by the central constructs in the Interconnected Model of Teacher Growth [4]. While this model focuses on the individual growth of the teacher, it is also a relevant perspective for reflecting on instructor engagement with course development. The model enables the identification of particular change and growth, giving recognition to the "idiosyncratic and individual nature of teacher professional growth" ([4], p.948). The model considers 4 analytic domains in which teachers function and grow: personal domain (knowledge, beliefs, and attitudes), the external domain (external sources of stimulus or information), the domain of practice (professional experimentation), and the domain of consequence (salient outcomes). According to Clarke \& Hollingsworth [4], one major value of the model is its capacity to stimulate research and development regarding change and is a powerful framework to support the analyses of those studying change (or growth) and the planning of those in educational contexts. For purposes of this analysis, we focused on the personal domain and the domain of consequence. Within the domain of consequence are the salient outcomes and for this analysis we defined those as the recommendations we would make to others as recommendations in some ways represent the sum of the experiential outcomes. Figure 1 is an operationalization of the two domains of the model salient to this analysis.

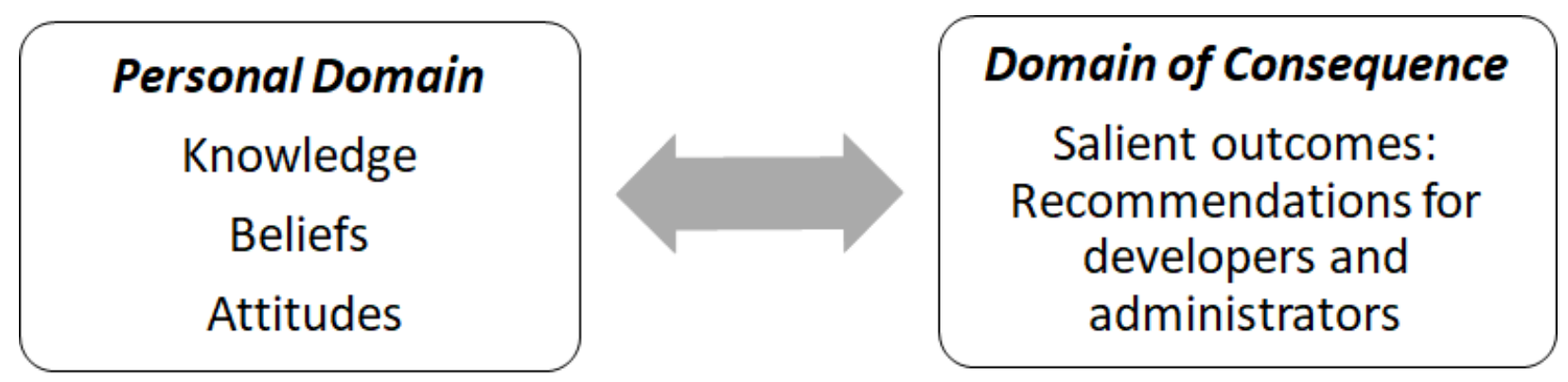

Figure 1: Salient elements of the Interconnected Model of Teacher Growth operationalized for this analysis [4].

This is an appropriate model to use for this work because in recent years the program has experienced considerable changes that align with the elements of this framework. In the personal domain, we have a large teaching team that brings variety in personal experiences. In the external domain, we have had changes in leadership at several levels and large changes in student enrollment numbers. In the domain of practice, we have experimented with different approaches with varying degrees of similarity and differences across individual sections of the course. In the domain of consequence, we have the need to continually improve our course and thus the development will go onward requiring recommendations for successful continued development engagement. This framework informed the development of our guidelines for autoethnography data collection and provided a frame of reference to conduct our analysis. 


\section{Methods}

Because we desired to understand the personal experience of course development and improvement within the context of a department, we designed our study as an autoethnography. Autoethnography is a research approach that blends the personal reflection of autobiographical writing with the examination of cultural relations, values, and beliefs that are part of ethnographies [5]. In contrast to social science research that has traditionally incorporated and recognized personal accounts [6], autoethnography is more intentional in this regard.

Duarte [7] describes the function of autoethnography: "As a reflexive genre of writing autoethnography situates the self within the context of a culture, sub-culture or group, and studies one's experience along with that of other members of the group" (p. 2). Consistent with this function, three of the five authors of this paper wrote autobiographical reflections on their course development experiences and we collectively analyzed them to yield collaborative findings. Realizing the need to further address the context, a fourth author wrote a reflection from the perspective of an administrator.

We took an analytic autoethnography approach [8] that is more focused on rational scholarly endeavors, as opposed to the evocative autoethnography approach more focused on emotions related to the phenomenon [5]. Two authors are engineering education research experts and three authors are professors of practice. This combination of author roles and purposes gives us a multi-faceted view that allows us to consider a broader range of cultures, values and beliefs than if the whole team served in the same capacity. As part of our authoethnographic process, we followed Anderson's [8] proposed characteristics of analytic autoethnography: (1) the authors are members of the group they are researching; (2) they engage in analytic reflexivity; (3) they are visible in their narratives; and (4) they engage in dialogue with other informants beyond "the self" (p. 378).

\section{Context of the Course}

In our context, this work was conducted on the required two-course sequence required for every engineering student when joining the University. The program provides an introduction to engineering knowledge and skills as well as an opportunity for over 2,000 students per year to explore the more than 15 different majors from which they can choose. On average, and across years, student demographics are similar to national averages for engineering programs. As students do not select a major until the end of the second semester, there is a mix of major interests in each section. Each section had 30-36 students with one instructor until Spring 2019, when section size was increased to 72, and instructors gained 10 hours of Graduate Teaching Assistant (GTA) support for each section. The number of sections taught by each individual instructor ranges from one to five depending on other job responsibilities. The total number of people teaching the two-course sequence each year also varies but is often more than a dozen.

The course sequence has been taught using several models of instruction over the years, ranging from an approach where all instructors teach from the same material using the same assignments to one where each individual instructor has nearly complete autonomy over what and how they 
teach throughout the semester. While each of these approaches had unique advantages and disadvantages, there was a desire among departmental leadership to create a degree of consistency throughout the sections while still allowing for instructors to take ownership over how, and to some extent, what was taught in the classroom while maintaining the courses as relevant and engaging for students.

In the new model, instructors teach from a common course structure and general scope for content and course assessment. Nevertheless, individual instructors have the opportunity to individualize their sections of the course. Meeting the purpose of the course and functioning within a structure that aligns all the actors (multiple teachers, multiple sections of the course, graduate teaching assistant support, undergraduate graders, etc.) required to support the number of students, can be challenging to continuous improvement and on-going course development processes. This model was developed and refined by a team of faculty who also taught the subject courses over several semesters.

\section{Participants}

The five total participants in this study include three professors of practice, a tenured faculty member who has administrative responsibility for the first year program, and a tenure-track faculty member who was previously a professor of practice in the same department. A professor of practice is defined as someone who has industry experience, and whose academic role is characterized by a predominantly teaching nature. Participant details are given in Table 1. Consistent with autoethnographical approaches, the research team emerged as one with a comment set of research questions and interests which we decided to collaboratively explore. Therefore, unlike some other modes of research, there was not a call for participants or formal response rate. 
Table 1. Description of participants backgrounds

\begin{tabular}{|l|c|l|l|}
\hline Name & $\begin{array}{c}\text { Years Teaching } \\
\text { in First Year } \\
\text { Program }\end{array}$ & \multicolumn{1}{|c|}{$\begin{array}{c}\text { Course development } \\
\text { role/responsibility }\end{array}$} & \multicolumn{1}{c|}{ Background } \\
\hline Ben & 2.5 & $\begin{array}{l}\text { First-semester segment } \\
\text { development team }\end{array}$ & $\begin{array}{l}\text { 4 years industry experience, 2 years at } \\
\text { current school as Instructor, } 0.5 \text { years as } \\
\text { Associate Professor of Practice }\end{array}$ \\
\hline Holly & $5+$ & $\begin{array}{l}\text { Original course developer } \\
\text { years ago, current program } \\
\text { administrator }\end{array}$ & $\begin{array}{l}\text { 12 years combined consulting and } \\
\text { industry experience, 11 years total at } \\
\text { current school, 4.5 years experience in } \\
\text { departmental administration }\end{array}$ \\
\hline Homero & $4+$ & $\begin{array}{l}\text { First and second semester } \\
\text { segment development teams }\end{array}$ & $\begin{array}{l}\text { 6 years industry experience, 15 years in } \\
\text { academia, 5 years experience in } \\
\text { University administration, 1 year at } \\
\text { current school as Associate Professor of } \\
\text { Practice, 1.5 years at current school as } \\
\text { Assistant Professor }\end{array}$ \\
\hline Matt & 2.5 & $\begin{array}{l}\text { First and second semester } \\
\text { segment development teams }\end{array}$ & $\begin{array}{l}\text { 8 years industry experience, 2.5 years } \\
\text { total at current school as Assistant } \\
\text { Professor of Practice' }\end{array}$ \\
\hline David & 1.5 & $\begin{array}{l}\text { Second-semester segment } \\
\text { development team }\end{array}$ & $\begin{array}{l}\text { 8 years industry experience, 1.5 years at } \\
\text { current school - 1 year as instructor, 0.5 } \\
\text { years as Associate Professor of Practice }\end{array}$ \\
\hline
\end{tabular}

\section{Data Collection and Analysis}

Individually, three of the participants, Matt, Ben and David, reflected on a series of questions informed by Interconnected Model of Teacher Growth [4]. This generated several-page written documents for each person. In addition to pragmatic questions about what worked well and what did not, we used the framework to make invisible or behind-the-scenes considerations visible. The reflection protocol is included as Appendix A. Although we considered the whole of the reflections, the most salient questions for this analysis include:

- What recommendations would you make to others embarking on a similar process?

- Where and how in this process did personal beliefs (yours or others) come into play (including previous personal experiences)?

Holly reflected on similar questions which were modified for the administrative perspective though the wording for the two salient questions used herein remained the same. It should be noted that Holly's reflection came after the first pass of collaborative analysis, though before final analysis, as it became evident that the reflection was needed to understand the lens she 
brought to the analysis process and her administrative role that set some context for the course development process.

For analysis, all members of the author team reviewed the individual reflections and we met as a group to discuss them. We started by listing the recommendations made by each participant and then connected them back to the personal experiences noted in other areas of the reflection. The notes from this session were translated to the textual story presented herein and Holly's reflective positionality added. Each participant reviewed the paper and had the opportunity to revise any aspects of their story in response to the collective story.

\section{Measures of Quality}

Walther et al. [9] suggest that researchers should consider research quality in terms of making the data and handling the data. In making the data, the research team followed approaches consistent with autoethnography, specifically Anderson [8]. Each person individually and critically reflected on their experiences and documented these reflections in narrative reports. In terms of handling the data, the research team used a protocol informed by theory in order to focus these reflections so they could be collectively and collaboratively evaluated. We worked collaboratively on the analysis to develop the results giving participants a chance to respond to the analysis process and findings as they emerged. The study secured ethical clearance from the Institutional Review Board (IRB).

\section{Limitations}

A limitation of this work is that one author, Holly, has administrative responsibility for the course sequence within the department. While this role was known when the collaboration emerged and we started the project, it may still have impacted participants' desire to reflect freely and openly specifically regarding challenges associated with work assigned by the administrator. Regardless, we believe we captured elements of our experience that can be meaningful to others. Future work could consider additional reflective activities to specifically elicit the degree to which this may have been a challenge.

\section{Results}

We organize our results to answer our research question, What are the individual, collective, and pragmatic outcomes from engaging faculty from a variety of backgrounds in on-going continual course development and improvement in a large first-year engineering program? The outcomes are the recommendations we would make and we have analyzed how they connect to personal experience. Therefore, we first present results person-by-person for those doing the course development to acknowledge the critical self-reflective intent of autoethnography. We then consider the patterns across the individual reflections, and weave in the administrator reflection, to understand these personal reflections in the collective context. Finally, we summarize high level recommendations that are essentially the pragmatic outcomes. 


\section{Individual Recommendations as Connected to Personal Experience}

David- In response to what recommendations he would have for others, David suggested matching expectations for the work with the time commitment needed/available to do the work. He also noted the importance of engaging in professional development related to teaching and course design "in order to build a knowledge base and the language needed to readily talk to others". Finally he noted working with others within and outside the department or College as a "critical support mechanism". These recommendations emerged from his newness to a formal teaching role in academia from an industry role, an awareness that he was entering a new field (engineering education from a more traditional engineering field), and the self-awareness of knowing he lacked teaching practice and pedagogical knowledge expertise. These recommendations also emerged from his fundamental way of functioning, "I tend to work best when I have a few colleagues with whom I can test my assumptions and ideas. Throughout this design process, I was examining my own concepts and designs with my colleagues, with students, and with my family and friends outside of the university."

Matt- In his response to recommendations for others, Matt noted the importance of not working alone and of "[f]inding a team of people who are committed to similar goals, but coming from different backgrounds". Matt also suggests considering the history of the course and how things came to be the way they are at present, "taking into account what the course did historically and people's appetite for change is important--if there were things that were being done well, think really critically before cutting them. If there were things that didn't align with the course as well as others but some people like doing them, try to leave some wiggle room for instructors to teach to their own passions." Finally, Matt highlighted a need to understand that it is an iterative process involving feedback, "Don't expect to get it right the first time, so be open to criticism (especially to criticism from those who come from different educational, teaching, professional, personal, etc. backgrounds)." Considering the personal domain of the teacher development model, these recommendations are consistent with Matt's prior experience working with clients, "Designing a course, fundamentally, isn't so different than designing a site for a new building. There are a lot of stakeholders with various views and often competing desires, the outcome is not necessarily clear other than some high-level goals, and you're working with a relatively small team of people on something that will influence a lot of people moving forward." They are also consistent with one of his fundamental beliefs, "I have also always firmly believed that people should have the opportunity for ownership of their own jobs to do their best, even if this means letting them make mistakes."

Ben- In writing his recommendations, Ben suggested starting at a high level and seeking broad open ended feedback. He said, "Ask everyone you can to tell you what they think the course is. Make your question vague. This will help check and understand your foundational assumptions. When you start at a high level, it's easier to see how the pieces fit, and easier to update them in ways that are in line with your goals." To support this broad data gathering he suggested system approaches to document and justify decisions, "Keep detailed notes, and write additional background and justification for what you are doing. This will help with assessment, and help with hand-off. It also helps you remember what you were thinking when you wrote an 
assignment or activity the way you did. You can provide small summaries (or more detailed summaries if so inclined) to the students, to help them understand why things are as they are." He offered examples of a change log and using a shared file management system. Ben's recommendations were driven by his personal experiences. In particular, Ben was inadvertently not given access to a significant portion of the core course materials until after the start of the semester, "I had a syllabus, a description of a modules-based course with details of major assignments, [and a few slide decks used by others in prior semesters]. The situation forced me to get creative and explore the subjects and what they meant to me." This is consistent with his recommendation to start with broad questions, challenge assumptions and document. His recommendations towards challenging assumptions are also consistent with having been in academia for a while, and having completed his $\mathrm{PhD}$ while teaching.

\section{Situating the stories within the collective context}

Across these three stories, the recommendations vary in direct connection to the personal experiences of Matt, Ben and David. Having space for such variation is consistent with the larger context in which the course development was occuring- a culture of faculty that teach individually but collectively, and a culture where different people have different opinions on what the course should be. From the administrator perspective, Holly's recommendations are consistent with this culture and the variability in Matt, Ben, Ben and David's reflections. First, Holly suggested that administrators set a goal for course development "that they can clearly communicate what it means and what purpose it serves." Holly's goal was "Consistency with Autonomy" such that students could expect similar experiences across multiple sections of the same course and with different instructors but faculty could have some individualized control over the course content and exercise individualized classroom practices. Holly also indicated that she did not have a specific goal for what the course content should include but rather wanted a "collective vision that is malleable enough to change with changing students and teachers in our program". Like Ben she saw value in understanding the opinions of many on the content. Similar to Matt, Holly recommends choosing "the right team to do the work and then trust and respect the people doing the work." These recommendations tie directly to Holly's beliefs that autonomy is important, to her own disdain for being told what to do, and having less experience actually teaching the course than the professors of practice (similar to David's experience in a new space). These recommendations also suggest an alignment between the course development and administrative perspectives where variability in approach to course development make sense given the lack of specified course content and a desire for course developer autonomy which create an environment for an open-ended problem. Such an approach is not without its challenges and we do not advocate that all departments adopt such an approach but rather advocate for considering factors beyond just course content and being mindful of alignment in expectations and experiences such that people can do course development work in ways that align with the experiences that drive them.

Also consistent across all three course developer reflections there is a prominence of the idea of "Don't do it alone," though it manifests in different ways. David notes that people within and outside his department provide an important support mechanism. Ben suggests talking with as many people as possible to develop an understanding of what the course even is. Matt focuses on 
making change but supporting the autonomy of the faculty stakeholder in the process that will be teaching the course. Taken collectively, these three approaches result in a comprehensive approach to interpersonal interaction in support of course development- receiving support, gathering stakeholder input, and empowering others.

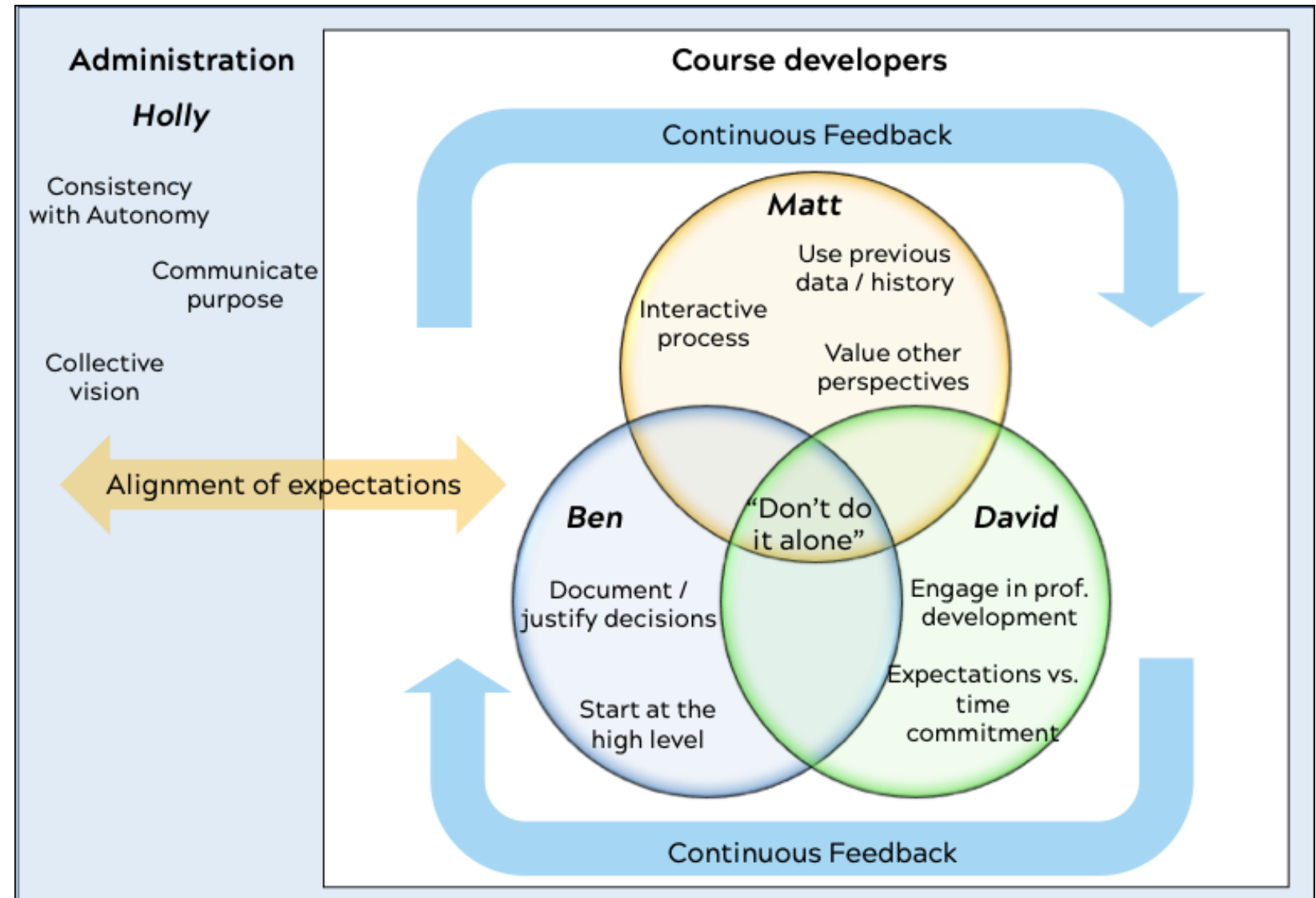

Figure 2. Summary of our findings

We also noted across all three course developer and the administrator reflections that no recommendations were made regarding gathering the student perspective in the salient questions for this analysis. In fact, there is only one reference to students, from Ben, and this is regarding justifying the course content to them, While all three people mentioned having drawn on the student perspective it was not explicitly mentioned here.

\section{Pragmatic Recommendations}

Finding that personal characteristics and experiences influence how professors of practice at one university approached course development in common first year course sequence in a large research University, we make the following high level pragmatic suggestions:

Build a course development team made up of people with different personal characteristics and backgrounds. Seeing that the approach each person in our project took had different foci, taking only one approach could exclude important elements or stakeholders from the process. A course 
development team might want to look at the three approaches described here and choose elements across the approaches that are consistent with their context.

In setting the scope of the course redesign project, consider the departmental culture. If the culture is perceived to be resistant to change, it may be necessary to make incremental changes at first or spend more time upfront getting buy-in on the need for substantial change. In this context, change-averse means holding fast to current or historical content or pedagogies or being unwilling to let go of what is personally important if it is not deemed collectively important.

Even in a change-supportive culture, support from departmental administration may be needed to clear the way so the team can focus on the course design work. Similar to open-mindedness regarding change, having a collegial nature in the department can support and facilitate change. While collegial can mean many things, in this case, we operationalize it to mean willingness to share ideas, willingness to let others shape/change individual ideas, and willingness to implement changes suggested by colleagues. A final consideration could be if the work is happening in a Department of Engineering Education. If so, recognizing what role, if any, formal education in educational methods will play may be important. If not, we suggest finding the appropriate support at the institution where the work is being developed. Most universities will have a center or group that supports instructional change and development, curricular initiatives, pedagogical change, and course transformation. Taking advantage of the experts in these topics we consider is a crucial aspect of being successful in the process.

Departmental resources should also be considered while setting the scope of course redesign projects. While course redevelopment may require funding for new materials, ensuring adequate time for those enacting the redevelopment is crucial. Having a course release to engage in this work will make a big difference in terms of what can reasonably be accomplished during the semester. When determining the time required for development, the starting point of the effort should be considered - i.e. are the developers working from an existing set of materials, or create an entirely new course? Both take time, but project outcome expectations may vary depending on the starting materials. Finally, the team needs to consider that it takes longer to develop materials that are ready to share with others than creating them for one's own use. The former requires a greater degree of formalizing, generalization, and articulation of purpose and function to be usable by others. Often, generalized materials will need to be personalized before implementation, even by those who originally created the materials.

\section{Discussion}

The purpose of this paper is to understand the individual, collective, and pragmatic outcomes from engaging faculty from a variety of backgrounds in on-going continual course development and improvement in a large first-year engineering program. Guskey [10] suggests that to optimize a process (e.g., course redesign) the understanding of that process from multiple perspectives is crucial. Our findings confirm the constructs proposed by Clarke \& Hollingsworth [4] model of teacher growth. The authors suggest that in teachers, change is identified with learning, and it is regarded as a natural and expected component of the professional activity. We consider that our recommendations are relevant because the actors involved in this research were 
learning and growing through a reflective process when they were undergoing a major transformation of the courses they teach.

We consider the personal and practice domains in Clarke \& Hollingsworth's [4] model of teacher growth was especially relevant to our findings. The personal domain focuses on teachers' attitudes, beliefs, and knowledge, and how those inform teachers' growth and change processes. The practice domain focuses on how teachers' previous professional experience shape their teaching views and the change processes they develop. We consider that our participants went through the process of curricular transformation, considering their personal attitudes, beliefs, and knowledge obtained from previous industry and personal experiences. We could evidence that depending on the previous experience and professional background, the participant was more or less focused on a different aspect of the process. For example, Matt valued the understanding that the process was iterative and might require several adjustments and trials before getting it "right", this aligns with his experiences as a Civil engineer where he interacted with multiple clients and recognized the importance of several iterations on his designs before satisfying the client. It is often cited in the literature that faculty members "teach the way they were taught" [11]-[14] this also means to recognize positive and negative experiences they had when they were students to inform the do's and don'ts of their teaching praxis. Our results align with this premise from the fact that professors of practice were bringing their self into the course development process not re-enacting the same or opposite as was acted on when they were students.

Similarly, we consider that some findings were aligned with the domain of consequence [4]. In this domain, change is framed within the inferences a teacher makes from their practices and experiences in the classroom. We were able to evince how some of the recommendations provided by the professors of practice were informed by their current experiences teaching the course. For example, Ben highlighted the importance of documenting decisions to keep track and remember the reasons behind the things we do. This is something that he has also been doing in his teaching practices when developing his own course materials. Finally, Clarke \& Hollingsworth explain the importance of planned professional development activities as part of the model for teacher growth. In the same line, David placed value on professional development and interacting with the experts when developing these processes.

One final point of discussion for this paper is the fact that the student perspective was not explicit in the results. We consider this to be an interesting finding that can make us more aware in the future and be more intentional about getting this perspective when doing course development. However, we consider that part of the reason why this was not prevalent in our discussion is because after teaching first-year engineering students we understand the difficult transition that students have when going to college. Hence, sometimes they have not had time to think about what they would expect or need from a first-year program, so we consider at that point it is more important to develop a program that can be more effective and engaging for them based on what many years of historical data and experiences can provide in shaping our understanding of what they need. We consider we do a good job of collecting students' feedback on individual classes rather than on doing it at the program level. 


\section{Conclusions and Future Work}

In conclusion, we found a clear connection between the personal domain and the domain of consequence in a course development process. Specifically, we found a connection between personal experiences and course development recommendations. We provide recommendations that can be used by practitioners, instructional designers, and administrators when going through a process of course redesign. Our recommendations not only apply to first-year engineering programs, but they can be adapted into any context.

We consider that these processes require administrative support in terms of recognizing the time not only to develop the redesign, but also to collect the data required to make the process effective. Similarly, it is important to align administrative expectations with course developers expectations. We consider finding a diverse team in terms of backgrounds and experiences that values multiple perspectives and establishes ongoing feedback processes. We also consider it relevant to understand that this is an iterative process that will require multiple attempts. It is important to identify allies that can support the process and are experts in educational research, pedagogy, or instructional design.

In terms of future work we would like to conduct research to better understand the role of students' feedback in course redesign processes. As mentioned before, despite us having multiple conversations about how important it is to consider the students' perspectives, and how we had the goal of making the redesign to have first year engineering programs more relevant and engaging for our students, this is something that did not emerge from our data. Hence, we consider we would like to explore this phenomenon better in the future.

\section{References}

[1] J. E. Froyd and G. J. Rogers, "Evolution and evaluation of an integrated, first-year curriculum," in Proceedings Frontiers in Education 1997 27th Annual Conference. Teaching and Learning in an Era of Change, 1997, vol. 2, pp. 1107-1113.

[2] C. E. Brawner, X. Chen, M. W. Ohland, and M. K. Orr, "The effect of matriculation practices and first-year engineering courses on engineering major selection," in 2013 IEEE Frontiers in Education Conference (FIE), 2013, pp. 1217-1223, doi:

10.1109/FIE.2013.6685023.

[3] H. V. Chang, Autoethnography as Method (illustrated edition.). California: Left Coast Press Inc, 2008.

[4] D. Clarke and H. Hollingsworth, "Elaborating a model of teacher professional growth," Teaching and teacher education, vol. 18, no. 8, pp. 947-967, 2002.

[5] C. Ellis and A. P. Bochner, Composing ethnography: Alternative forms of qualitative writing, vol. 1. Rowman Altamira, 1996.

[6] N. K. Denzin, “Analytic autoethnography, or déjà vu all over again,” Journal of contemporary ethnography, vol. 35, no. 4, pp. 419-428, 2006.

[7] F. Duarte, "Using autoethnography in the scholarship of teaching and learning: reflective practice from "the other side of the mirror,"' 2007.

[8] L. Anderson, "Analytic autoethnography," Journal of contemporary ethnography, vol. 35, 
no. 4, pp. 373-395, 2006.

[9] J. Walther, N. W. Sochacka, and N. N. Kellam, "Quality in interpretive engineering education research: Reflections on an example study," Journal of Engineering Education, vol. 102, no. 4, pp. 626-659, 2013.

[10] T. R. Guskey and M. Huberman, Professional development in education: New paradigms and practices. ERIC, 1995.

[11] A. Oleson and M. T. Hora, "Teaching the way they were taught? Revisiting the sources of teaching knowledge and the role of prior experience in shaping faculty teaching practices," Higher education, vol. 68, no. 1, pp. 29-45, 2014.

[12] S. E. Cox, "Perceptions and Influences Behind Teaching Practices: Do Teachers Teach as They Were Taught?," 2014.

[13] J. Beegle and D. Coffee, "Accounting instructors' perceptions of how they teach versus how they were taught," Journal of education for Business, vol. 67, no. 2, pp. 90-94, 1991.

[14] T. Hopper, "PE teachers teach the way they were taught: asset or handicap?," Physical \& Health Education Journal, vol. 66, no. 1, p. 47, 2000. 


\section{Appendix A: Autoethnography Reflection Guide}

Each project team member will write a reflection addressing questions similar to the following. Note that a final protocol has not been developed but these questions reflect the general scope of the reflection.

\section{$\underline{\text { Reflection on pragmatic aspects of course development: }}$}

How did you approach course development? What worked and what did not work in your approach? How do you know?

What are the principal changes that you made in the course? What worked? What did not work? How do you know?

What were some of the principal challenges that you encountered in course development? How did you address these challenges?

What recommendations would you make to others embarking on a similar process?

\section{Deeper Reflection on the Experience:}

Where and how in this process did personal beliefs (yours or others) come into play (including previous personal experiences)?

What departmental and university factors contributed to course development decisions (positive and negative)?

What external factors associated with a broader context contributed to course development decisions (positive and negative)?

What, if any, experimentation was involved (either prior to or during the semester)?

What salient outcomes were considered for students, faculty and other stakeholders? 